\title{
Mapping the column density and dust temperature structure of IRDCs with Herschel ${ }^{\star}$
}

\author{
N. Peretto ${ }^{1,2}$, G. A. Fuller ${ }^{1}$, R. Plume ${ }^{3}$, L. D. Anderson ${ }^{4}$, J. Bally ${ }^{5}$, C. Battersby ${ }^{5}$, M. T. Beltran ${ }^{6}$, J.-P. Bernard ${ }^{8}$, \\ L. Calzoletti ${ }^{12}$, A. M. DiGiorgio ${ }^{10}$, F. Faustini ${ }^{12}$, J. M. Kirk ${ }^{7}$, C. Lenfestey ${ }^{1}$, D. Marshall ${ }^{8}$, P. Martin ${ }^{9}$, \\ S. Molinari ${ }^{10}$, L. Montier ${ }^{8}$, F. Motte ${ }^{2}$, I. Ristorcelli ${ }^{8}$, J. A. Rodón ${ }^{4}$, H. A. Smith ${ }^{11}$, A. Traficante $^{12}$, \\ M. Veneziani ${ }^{12}$, D. Ward-Thompson ${ }^{7}$, and L. Wilcock ${ }^{7}$
}

(Affiliations are available in the online edition)

Received 31 March 2010 / Accepted 30 April 2010

\begin{abstract}
Infrared dark clouds (IRDCs) are cold and dense reservoirs of gas potentially available to form stars. Many of these clouds are likely to be pristine structures representing the initial conditions for star formation. The study presented here aims to construct and analyze accurate column density and dust temperature maps of IRDCs by using the first Herschel data from the Hi-GAL galactic plane survey. These fundamental quantities, are essential for understanding processes such as fragmentation in the early stages of the formation of stars in molecular clouds. We have developed a simple pixel-by-pixel SED fitting method, which accounts for the background emission. By fitting a grey-body function at each position, we recover the spatial variations in both the dust column density and temperature within the IRDCs. This method is applied to a sample of 22 IRDCs exhibiting a range of angular sizes and peak column densities. Our analysis shows that the dust temperature decreases significantly within IRDCs, from background temperatures of 20-30 K to minimum temperatures of 8-15 K within the clouds, showing that dense molecular clouds are not isothermal. Temperature gradients have most likely an important impact on the fragmentation of IRDCs. Local temperature minima are strongly correlated with column density peaks, which in a few cases reach $N_{\mathrm{H}_{2}}=1 \times 10^{23} \mathrm{~cm}^{-2}$, identifying these clouds as candidate massive prestellar cores. Applying this technique to the full Hi-GAL data set will provide important constraints on the fragmentation and thermal properties of IRDCs, and help identify hundreds of massive prestellar core candidates.
\end{abstract}

Key words. stars: formation - ISM: clouds

\section{Introduction}

One of the major issues in star formation is understanding the physical conditions, including the temperature and density structure, of the material out of which stars will form. While fundamental, these quantities are difficult to determine. These regions are cold and therefore radiate energy away through dust at the far infrared/submillimeter wavelengths, a frequency range barely accessible from the ground because of the opacity of the atmosphere.

For the first time, a high angular resolution view of the Universe from 70 to $500 \mu \mathrm{m}$ is now becoming available thanks to the ESA Herschel Space Observatory (Pilbratt et al. 2010). The frequency range provided by its two photometry instruments PACS (Poglitsch et al. 2010) and SPIRE (Griffin et al. 2010) perfectly matches the emission peaks of young stellar objects and prestellar cores, the direct progenitors of stars. It is now possible to construct well constrained SEDs for these objects, and therefore determine the spatial distributions of the temperature and column density towards a wide range of different objects.

Hi-GAL is the open time key program to observe the inner part $\left(|l| \leq 60^{\circ} ;|b| \leq 1^{\circ}\right)$ of the Galactic plane with Herschel (Molinari et al. 2010a). The sensitivity of this survey will

* Herschel is an ESA space observatory with science instruments provided by European-led Principal Investigators consortia and with important participation from NASA. allow the detection of $10 M_{\odot}$ molecular clouds at a distance of $4 \mathrm{kpc}$. A large number of diverse studies will result from this survey covering scientific themes such as evolved stars, diffuse interstellar medium, and star formation. In this Letter, we present the first Herschel study analyzing the temperature and column density structure of infrared dark clouds (IRDCs). These objects are reservoirs of cold, dense molecular gas seen in extinction in the mid-infrared against the strong background emission of the Galactic plane (Perault et al. 1996; Rathborne et al. 2006, Fig. 1). This gas is only slightly processed by current star formation activity and most likely still contains the initial conditions of star formation imprinted within them. Their study can therefore illuminate the earliest stages of star formation. In Sect. 2 of this paper, we describe the data, Sect. 3 focuses on the background properties. Section 4 presents the first results, while Sects. 5 and 6 summarize the discussion and conclusions of the paper, respectively.

\section{Data}

This analysis uses data from the Hi-GAL science demonstration phase (SDP) which consists of two tiles of $\sim 2.2^{\circ} \times 2.2^{\circ}$ centered on $l=30^{\circ}$ and $l=59^{\circ}$. For each tile, the parallel mode of observations provides PACS $70 / 160 \mu \mathrm{m}$ and SPIRE $250 / 350 / 500 \mu \mathrm{m}$ images. The SDP data were reduced using the ROMAGAL data reduction software (see Molinari et al. 2010b, for a complete description). The recommended calibration factors and offsets 


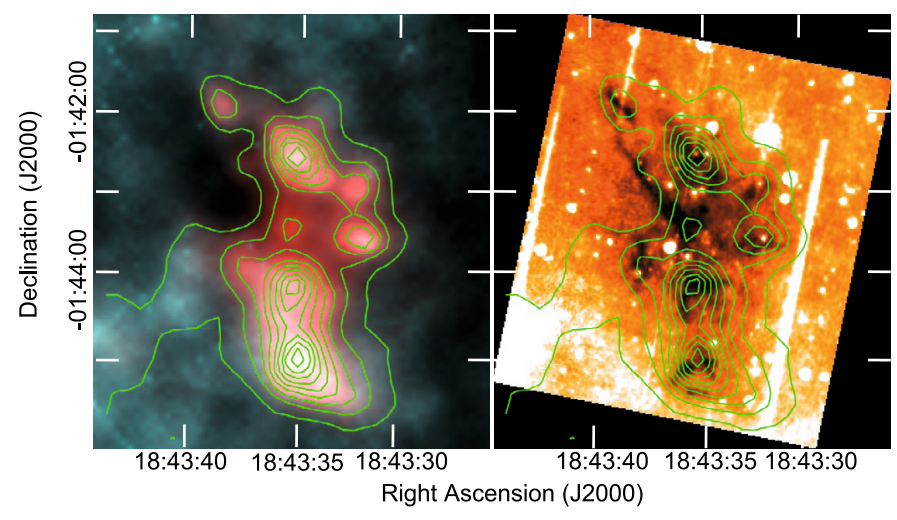

Fig. 1. Left: two color image of one of the 22 IRDCs analyzed here. The blue is the $160 \mu \mathrm{m}$ emission while the red and the contours show the $250 \mu \mathrm{m}$. Right: Spitzer $8 \mu \mathrm{m}$ image of the same IRDC on which we have overlaid the Herschel $250 \mu \mathrm{m}$ contours.

were applied to the data to set the zero flux level Bernard et al. (2010).

In total, Peretto \& Fuller (2009) catalogued 450 Spitzer IRDCs in the 2 tiles of the Hi-GAL SDP fields, $75 \%$ of these being in the $l=30^{\circ}$ tile. Checking each of these clouds by eye, we identified 80 to $90 \%$ of these IRDCs seen in emission with Herschel at long wavelengths, confirming that these are real clouds, not caused by artifacts in the Spitzer $8 \mu \mathrm{m}$ background. For this study, we selected a subsample of 22 IRDCs (see Fig. 1), with sizes ranging from $40^{\prime \prime}$ to $180^{\prime \prime}$, all large enough to contain at least one $500 \mu \mathrm{m}$ beam (i.e., $36^{\prime \prime}$ ) and with a column density peak derived from extinction such as $N_{\mathrm{H}_{2}}^{\text {peak }} \geq 3 \times 10^{22} \mathrm{~cm}^{-2}$, which is high enough to clearly stand out of the Hi-GAL data background. All images were resampled to a common pixel size of $2^{\prime \prime}$. We excluded the W43 region because of its complex background structure (Bally et al. 2010). Our selected sample is clearly biased towards rather large and massive IRDCs.

\section{Background emission towards the IRDC}

The equation governing the emission $I_{v}$ towards an IRDC in the optically thin case is:

$$
\begin{aligned}
I_{v} & =B_{v}\left(T_{\mathrm{d}}^{\mathrm{IRDC}}\right)\left(1-\mathrm{e}^{-\tau_{v}^{\mathrm{IRDC}}}\right)+I_{v}^{\mathrm{back}} \mathrm{e}^{-\tau_{v}^{\mathrm{IRDC}}}+I_{v}^{\text {fore }} \\
& =B_{v}\left(T_{\mathrm{d}}^{\mathrm{IRDC}}\right) \times \tau_{v}^{\mathrm{IRDC}}+I_{v}^{\mathrm{bg}},
\end{aligned}
$$

where $B_{v}\left(T_{\mathrm{d}}^{\mathrm{IRDC}}\right)$ is the Planck function at the temperature of the IRDC averaged along the line of sight, $\tau_{v}^{\mathrm{IRDC}}$ is the opacity of the IRDC at the corresponding frequency, and $I_{v}^{\mathrm{bg}}$ is the combined background and foreground emission, which is approximately independent of $\tau_{v}^{\mathrm{IRDC}}$ in the optically thin limit. Equation (2) shows that to calculate the opacity and temperature for images at a range of wavelengths, the background emission $I_{v}^{\text {bg }}$ at each wavelength needs first to be determined.

The full images show that a significant fraction of the total flux is from large-scale extended emission (Molinari et al. 2010b). It is important to account for this emission to reliably map the IRDC emission because fluctuations in this background could otherwise be interpreted as structure in the IRDC. However defining the background structure is not trivial. Since

\footnotetext{
1 These sizes are those derived from the extinction and given in Peretto \& Fuller (2009). The sources are systematically larger in emission because the Hi-GAL data probes lower column density material.
}

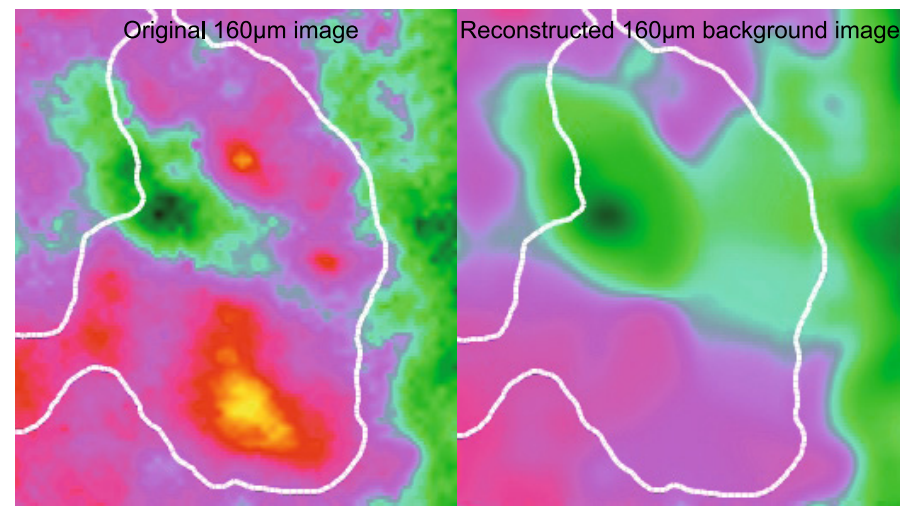

Fig. 2. Comparison of the original $160 \mu \mathrm{m}$ image left with the corresponding reconstructed background image right. The intensity scale is the same for both images. The reconstructed background image has been smoothed to $36^{\prime \prime}$, the common resolution used in for the SED fitting (cf. Sects. 4 and 5). The contour corresponds to the limit between IRDC and background derived from the $500 \mu \mathrm{m}$ data.

the cirrus noise decreases towards longer wavelengths (Gautier et al. 1992), we used the $500 \mu \mathrm{m}$ image to define the boundaries of each IRDC. (The $350 \mu \mathrm{m}$ data, which are very similar but have higherr angular resolution, were also used to confirm the boundary of each IRDC.) The pixel flux distribution at $500 \mu \mathrm{m}$ toward IRDCs shows in most cases a well defined peak at low fluxes and extended tail at larger fluxes corresponding to the IRDC. The level to separate the background and IRDC was chosen to be one standard deviation above the peak value, the standard deviation being calculated from only those pixels below the peak. This procedure allows us to identify the pixels in the image associated with the IRDC, and those pixels containing only background emission (Fig. 2). This procedure works well, even for small IRDCs, provided there are sufficient pixels to characterize the background emission.

To take the background fluctuations into account, we reconstructed at all wavelengths background images by interpolating the background pixels at the position of the IRDC pixels. After experimenting with various interpolation methods, we decided to use an interpolation based on the nearest neighbors. However, the interpolation method has little impact on the global physical properties since flux uncertainties are dominated by cirrus noise at short wavelengths (cf. next paragraph) and calibration uncertainties at longer wavelengths. In Fig. 2, we show the reconstructed background images of the IRDC at $160 \mu \mathrm{m}$ as well as the original image on the same intensity scale. The most significant feature of this background image is the important decrease in intensity on the left part of the image (as also seen at $250 \mu \mathrm{m}$; Fig. 1), which is recovered well on the reconstructed background image.

Uncertainties in these background images are obviously higher at the center of the IRDCs, where pixels are further away from a background pixel. To estimate this uncertainty, we computed a two point flux difference for all background pixels as a function of their angular separation, and this at each wavelength (Fig. 3). As observed for all IRDCs, the uncertainties are much higher at $160 \mu \mathrm{m}$ than at $500 \mu \mathrm{m}$, justifying our choice of choosing the latter for defining the IRDC boundaries. When the uncertainties computed this way are higher than the $20 \%$ calibration uncertainty, we used them on the flux measurement when performing pixel by pixel SED fitting (cf. Sect. 4).

The SED of the background images were fitted to determine the properties of the dust responsible for the background 


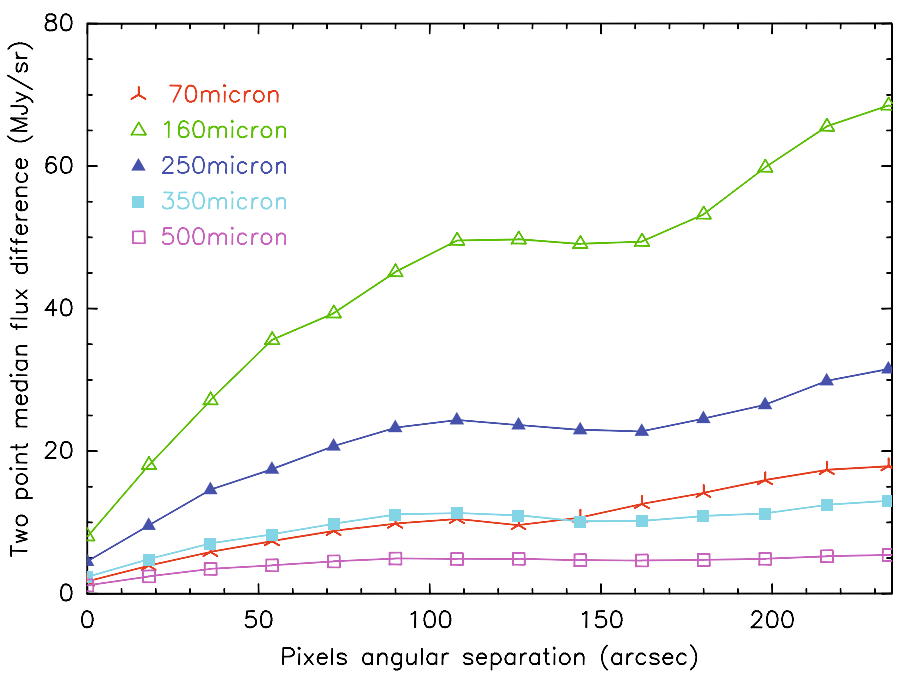

Fig. 3. Evolution of the flux difference between 2 background-only pixels as a function of their angular separation for the IRDC shown in Fig. 1 (after smoothing to a common $36^{\prime \prime}$ resolution). This clearly shows that the background is far more structured at $160 \mu \mathrm{m}$ than at $500 \mu \mathrm{m}$, justifying our choice of using the latter to define the boundaries of the IRDCs.

emission (see Fig. 4). With 5 data points per pixel (from 70 to $500 \mu \mathrm{m}$ ), we can constrain, in addition to opacity and dust temperature, the spectral index $\beta$ of the specific dust opacity law, where $\tau_{v} \propto \nu^{\beta}$. Doing so, we find that the background dust temperature varies from $\sim 20$ up to $\sim 30 \mathrm{~K}$ depending on the position of the IRDC in the Galactic plane, in agreement with Bernard et al. (2010), while $\beta$ varies from 1.6 up to 2.3 , consistent with Boulanger et al. (1996).

\section{Column density and dust temperature maps of IRDCs}

The reconstructed background images provide the $I_{v}^{\mathrm{bg}}$ term in Eq. (2) for each pixel of the IRDC. The values of $\tau_{v}^{\mathrm{IRDC}}$ and $T_{\mathrm{d}}^{\mathrm{IRDC}}$ at each pixel are then determined by minimizing the $\chi^{2}$ between the observed fluxes and those given by Eq. (2). For this purpose, we used the MPFITS package (Markwardt 2009), as for the background SED fitting.

The SED fitting for IRDC pixels was performed using 4 data points per pixel, smoothed to the same resolution of $36^{\prime \prime}$. The $70 \mu \mathrm{m}$ point was excluded since at this wavelength the optically thin assumption is not valid, the IRDCs are usually seen in absorption and, therefore, the optically thin assumption is invalid (see Sect. 5 for a discussion about $70 \mu \mathrm{m}$ point like sources). Because of the small number of data points for each fit, we fixed $\beta$ leaving two free parameters. Adopting the same value of $\beta=2$ for all IRDCs, we calculated dust temperature maps (cf. Figs. 4 and 5). The first important feature of the map is that the dust temperature is non-uniform, ranging from $10 \mathrm{~K}$ for the coldest central cores, up to $22 \mathrm{~K}$, consistent with the inferred background temperature.

To obtain a superior angular resolution, we iterated the SED fitting excluding this time the $500 \mu \mathrm{m}$ point, and using the dust temperature map and column density map calculated in the first iteration as an input parameter to the fit of the second iteration. Doing so, the temperature map changes little, while the column density map appears more structured. This is not really surprising because the temperature is predominantly constrained by the shortest wavelength data, toward the peak emission and on the

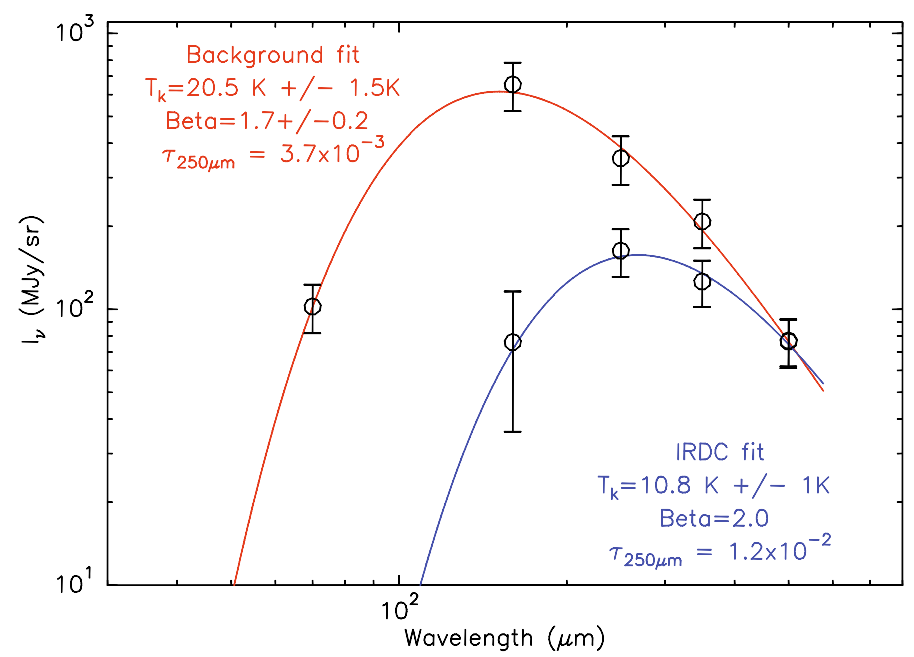

Fig. 4. SED fit of a background-only pixel of the IRDC shown in Fig. 1 (red) and the pixel at the column density peak (blue). The solid lines are the best fit to the data points. The uncertainties in the background are the $20 \%$ calibration ones, while for the IRDC we took the larger of the calibration uncertainty and the ones described by Fig. 3 .

exponential part of the Planck function. Given this, we directly used the $36^{\prime \prime}$ resolution temperature map in combination with the $250 \mu \mathrm{m}$ images to obtain $18^{\prime \prime}$ resolution column density maps. The resulting column density map for the IRDC shown in Fig. 1 is shown as contours in Fig. 5. We assumed a normalization of the specific dust opacity law of $0.12 \mathrm{~cm}^{2} \mathrm{~g}^{-1}$ at $250 \mu \mathrm{m}$, adopting the thin ice mantle dust grain models of Ossenkopf \& Henning (1994) at a density $n=10^{5} \mathrm{~cm}^{-3}$. Uncertainties in this normalization and $\beta$ directly implies at least a factor of 2 of uncertainty in the column density and a couple of degrees for the temperatures. It is possible that $\beta$ decreases systematically towards the inner region of IRDCs because of dust coagulation (Ossenkopf \& Henning 1994). However, even a decrease from $\beta=2$ to $\beta=1.5$ increases the minimum temperature by only $\sim 2 \mathrm{~K}$, which is insufficient to reverse the observed trends discussed in the next section.

We can already note several important points here. First, the strongest peaks at $250 \mu \mathrm{m}$ seen in Fig. 1 are not the strongest column density peaks. Dust temperature variations have an important role in determining the emission. Most of the column density peaks are also correlated with local temperature minima, although there is not a one to one correlation. The 3D structure of the cloud is probably responsible for the apparent projection offsets.

\section{Discussion}

The most striking feature of our dust temperature maps is the non-isothermal nature of IRDCs. In Fig. 6, we plot the massweighted dust temperature versus the average $\mathrm{H}_{2}$ column density where the blue symbols correspond to the 22 analyzed IRDCs, while the red symbols correspond to the dust temperature and $\mathrm{H}_{2}$ column density peaks we visually identified within the IRDCs (for instance 6, peaks are identified in the IRDC of Fig. 5). While the median mass-weighted temperature is around $15 \mathrm{~K}$, we see that it can be as low as $8 \mathrm{~K}$ in the inner part of IRDCs. Knowing that background temperature lies in a 20 to $30 \mathrm{~K}$ range, this means that the temperature decreases by 10 to $15 \mathrm{~K}$ from edge to center. Therefore, the dust temperature in dense molecular clouds is highly non-uniform. 


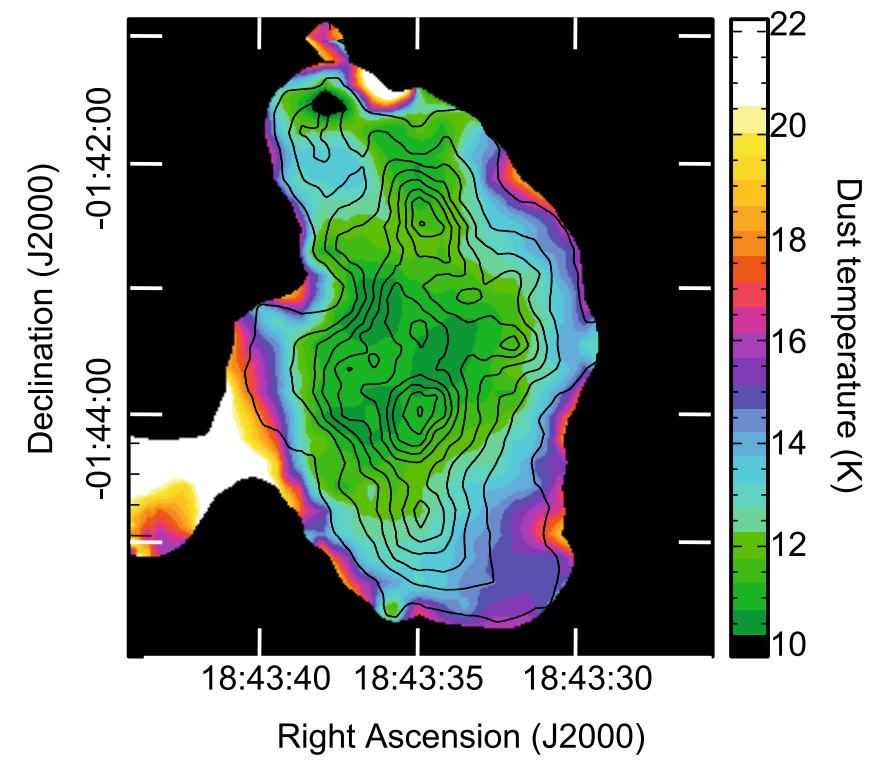

Fig. 5. Dust temperature map (color scale) for the IRDC shown in Fig. 1. The contours represent the $\mathrm{H}_{2}$ column density of this cloud, from $1 \times$ $10^{21}$ to $3.5 \times 10^{22} \mathrm{~cm}^{-2}$. We see that the strongest peaks in the $250 \mu \mathrm{m}$ image of Fig. 1 are not the strongest column density peaks.

Temperature and its fluctuations are fundamental parameters for fragmentation in molecular clouds. Hydrodynamical simulations including radiative transfer (Bate 2009; Krumholz et al. 2010) showed that protostellar heating could significantly reduce fragmentation on small scales. The picture we can draw from the study presented here suggests that IRDCs in the Galactic plane probably form from warm molecular gas with a temperature of $\sim 20 \mathrm{~K}$, which cools efficiently to $10 \mathrm{~K}$. On the other hand, in the previously mentioned simulations temperature gradients only develop once the first protostars form, in contrast to what we found here.

The filled symbols in Fig. 6 correspond to IRDCs (blue) or column density peaks (red) that contain at least one $70 \mu \mathrm{m}$ pointlike source. We can see that two thirds of the IRDCs and one third of the column density peaks are currently forming stars, consistent with the statistics from Spitzer observations of a large sample of IRDCs (Peretto \& Fuller, in prep.). For these starforming cores, the column density and temperature are probably overestimated and underestimated, respectively, since we did not take into account the data points at wavelengths below $100 \mu \mathrm{m}$. However, since the longest wavelengths have more weight in the SED fitting (due to their lower uncertainties) the column density of the cold gas should not be affected too much. Another interesting point is that when extrapolating the linear relation found by Dunham et al. (2008) between the $70 \mu \mathrm{m}$ flux of low mass protostars and their bolometric luminosity to the $70 \mu \mathrm{m}$ sources we identified here, we find that their bolometric luminosity lies somewhere between a few $1000 L_{\odot}$ and a few $10000 L_{\odot}$ depending on the source and their distance (typically between 3 and $6 \mathrm{kpc}$ ). This makes these sources excellent high-mass protostellar candidates.

Finally, Fig. 6 clearly shows high column density fragments with no $70 \mu \mathrm{m}$ point-like sources within them. These are interesting objects for high-resolution follow-up studies since they might well be the birth places of massive stars, before any star

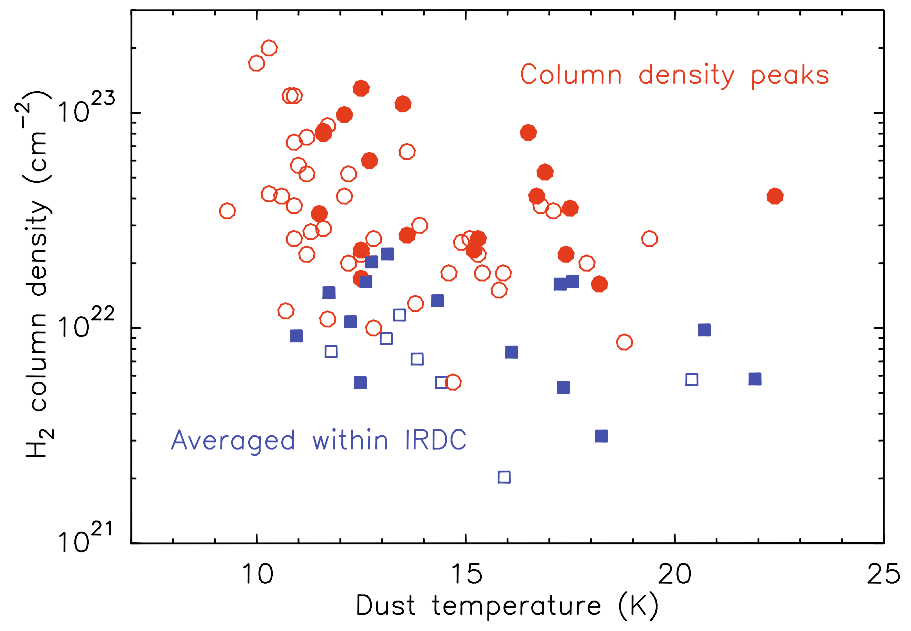

Fig. 6. Dust temperature versus column density plot. The blue square symbols show the average column density and mass weighted temperature for the 22 IRDCs analyzed. The red circular symbols show the temperature and column density at the column density peaks within the IRDCs. The filled symbols correspond to IRDCs with at least one $70 \mu \mathrm{m}$ point-like source, i.e., star-forming IRDC/peaks.

formation activity has started. Extrapolated to the entire Spitzer IRDC catalog of Peretto \& Fuller (2009), we expect to find a few hundred similar starless high column density cores in the full $\mathrm{Hi}-\mathrm{GAL}$ dataset.

\section{Conclusions}

We have developed a technique to obtain reliable temperature and column density maps of IRDCs using the first Herschel data from the Hi-GAL open-time key project. We have demonstrated our method for a small sample of massive IRDCs by showing that they are non-isothermal but have a definite temperature structure, their inner regions being cooler than their edges. This temperature structure probably has an important impact on the fragmentation of the cloud in the early stages of its evolution. We also identified a number of high column density peaks, reaching values of $10^{23} \mathrm{~cm}^{-2}$, which are most likely to be the birth sites of massive stars. The full Hi-GAL dataset is expected to contain several hundreds of these sources.

\section{References}

Bally, J., Anderson, L. D., Battersby, C., et al. 2010, A\&A, 518, L90 Bate, M. R. 2009, MNRAS, 392, 1363

Bernard, J.-Ph., Paradis, D., Marshall, D., et al. 2010, A\&A, 518, L88 Boulanger, F., Abergel, A., Bernard, J., et al. 1996, A\&A, 312, 256 Dunham, M. M., Crapsi, A., Evans, II, N. J., et al. 2008, ApJS, 179, 249

Gautier, III, T. N., Boulanger, F., Perault, M., \& Puget, J. L. 1992, AJ, 103, 1313 Griffin, M. J., Abergel, A., Abreu, A., et al. 2010, A\&A, 518, L3

Krumholz, M. R., Cunningham, A. J., Klein, R. I., \& McKee, C. F. 2010, ApJ, 713,1120

Markwardt, C. B. 2009, in ASP Conf. Ser. 411, ed. D. A. Bohlender, D. Durand, \& P. Dowler, 251

Molinari, S., Swinyard, B., Bally, J., et al. 2010a, PASP, 122, 314

Molinari, S., Swinyard, B., Bally, J., et al. 2010b, A\&A, 518, L100 Ossenkopf, V., \& Henning, T. 1994, A\&A, 291, 943

Perault, M., Omont, A., Simon, G., et al. 1996, A\&A, 315, L165

Peretto, N., \& Fuller, G. A. 2009, A\&A, 505, 405

Pilbratt, G. L., Riedinger, J. R., Passvogel, T., et al. 2010, A\&A, 518, L1

Poglitsch, A., Waelkens, C., Geis, N., et al. 2010, A\&A, 518, L2

Rathborne, J. M., Jackson, J. M., \& Simon, R. 2006, ApJ, 641, 389

Page 5 is available in the electronic edition of the journal at http: //www . aanda . org 
1 Jodrell Bank Centre for Astrophysics, School of Physics and Astronomy, The University of Manchester, Manchester, M13 9PL, UK

e-mail: nicolas.peretto@manchester.ac.uk

2 Laboratoire AIM, CEA/DSM-CNRS-Université Paris Diderot, IRFU/Service d'Astrophysique, C.E. Saclay, Orme des merisiers, 91191 Gif-sur-Yvette, France

3 Department of Physics \& Astronomy, University of Calgary, Canada

${ }^{4}$ Laboratoire d'Astrophysique de Marseille, UMR 6110 CNRS \& Université de Provence, France

5 Center for Astrophysics and Space Astronomy, University of Colorado, Boulder, USA

6 INAF, Osservatorio Astrofisico di Arcetri, Largo E. Fermi 5, 50125 Firenze, Italy
7 School of Physics \& Astronomy, Cardiff University, Queens Buildings, The parade, Cardiff, CF24 3AA, UK

${ }^{8}$ Centre d'Études Spatiales des Rayonnements, 9 Avenue Colonel Roche, 31022 Toulouse, France

9 INAF-Instituto Fisica Spazio Interplanetario, via Fosso del Cavaliere 100, 00133 Roma, Italy

10 Department of Astronomy \& Astrophysics, University of Toronto, Toronto, Canada

11 Harvard-Smithosonian Center for Astrophysics, 60 Garden Street, Cambridge, MA 02138, USA

12 Dipartimento di Fisica, università di Roma 2, Tor vergata, Rome, Italy

13 ASI Science Data Center, 000444, Frascati (Rome), Italy 